\title{
Intra-cluster correlation coefficients in adults with diabetes in primary care practices: the Vermont Diabetes Information System field survey
}

\author{
Benjamin Littenberg* and Charles D MacLean
}

Address: General Internal Medicine, University of Vermont, 371 Pearl Street, Burlington, VT, 05401, USA

Email: Benjamin Littenberg* - benjamin.littenberg@vtmednet.org; Charles D MacLean - charles.maclean@vtmednet.org

* Corresponding author

Published: 03 May 2006

BMC Medical Research Methodology 2006, 6:20 doi:10.1 186/147I-2288-6-20

This article is available from: http://www.biomedcentral.com/I47I-2288/6/20

(C) 2006 Littenberg and MacLean; licensee BioMed Central Ltd.

This is an Open Access article distributed under the terms of the Creative Commons Attribution License (http://creativecommons.org/licenses/by/2.0), which permits unrestricted use, distribution, and reproduction in any medium, provided the original work is properly cited.

\begin{abstract}
Background: Proper estimation of sample size requirements for cluster-based studies requires estimates of the intra-cluster correlation coefficient (ICC) for the variables of interest.

Methods: We calculated the ICC for II 2 variables measured as part of the Vermont Diabetes Information System, a cluster-randomized study of adults with diabetes from 73 primary care practices (the clusters) in Vermont and surrounding areas.

Results: ICCs varied widely around a median value of 0.0185 (Inter-quartile range: $0.006,0.037$ ). Some characteristics (such as the proportion having a recent creatinine measurement) were highly associated with the practice $(I C C=0.288$ ), while others (prevalence of some comorbidities and complications and certain aspects of quality of life) varied much more across patients with only small correlation within practices (ICC $<0.00 I)$.
\end{abstract}

Conclusion: The ICC values reported here may be useful in designing future studies that use clustered sampling from primary care practices.

\section{Background}

Multi-level or clustered sampling designs are increasingly deployed in medical and health care surveys. In these designs, clusters are identified (e.g. medical practices) and then subjects (e.g. patients) are sampled from each cluster. The analysis and sample size estimation for such designs must take the clustering into account or the resultant significance tests ( $P$ values) and confidence intervals will be in error [1]. Generally, failure to account for clustering leads to nominal confidence intervals that are too narrow and to $P$ values that are too small. To the extent that patient characteristics are independent of cluster, the effective sample size will be close to the number of individual subjects studied. If the subject characteristics are highly associated within clusters, the effective sample size approaches the number of clusters. In the extreme case, if all the subjects within a cluster are identical, there is no advantage to measuring more than one subject per cluster.

To estimate statistical power or required sample size in a study based on simple random sampling or allocation, one requires an estimate of the minimal important effect and (for continuous measures) the standard deviation of the outcome in the population studied. For clustered designs, however, one must also inflate the sample size to account for the clustering effect. The design effect, sometimes referred to as the variance inflation factor, is a function of the extent of correlation within clusters, the 
intraclass (or intra-cluster) correlation coefficient (ICC). Unfortunately, pre-study estimates of ICC are difficult to come by and obtaining them constitutes "the main difficulty in calculating sample size for cluster randomized studies"

Several groups have published estimates of ICCs for various patient characteristics observed in large surveys of patients clustered within primary care or general practices from around the world [3-6]. Here we expand their estimates to include those derived from a survey of adults with diabetes clustered within primary care practices in the northeast United States.

\section{Methods}

This study was part of a larger project, the Vermont Diabetes Information System (VDIS), a cluster-randomized trial of a laboratory-based diabetes decision support system in a region-wide sample of 8808 adults with diabetes from 73 Primary Care practices in Vermont and nearby parts of the United States [7]. Primary care in these predominantly rural practices is provided by General Internists, Family Physicians, Physician Assistants, and Nurse Practitioners who provide the bulk of long-term care for these and other patients. There are few diabetes specialists in the region and most diabetes care is provided in the practices. All 119 eligible primary care practices near the thirteen participating hospitals were invited to participate [7]. The participating practices range in size from one provider (in 41 practices) to two practices with six providers each.

A field survey targeted at a sub-sample of subjects was designed to provide a better understanding of the nonlaboratory features of the patients before intervention. Field survey subjects were selected at random from the patients participating in the VDIS and invited by telephone to participate in an in-home interview. Patient names were randomly sorted and patients contacted until a sample of approximately $15 \%$ of the patients from each practice agreed to an interview. We attempted to contact 4,209 patients and reached $1,576(37 \%)$. Of these, 1,006 (64\%) agreed to be interviewed.

Subjects who agreed were mailed a questionnaire and were scheduled for an interview by a trained field interviewer. During the visit, the interviewer reviewed any missing or ambiguous questionnaire items. If necessary, the interviewer read the questions aloud for subjects and recorded their responses for them. Then the interviewer measured the subject as described below and administered a few more instruments that were not included in the questionnaire. The interviews took place during the baseline phase of the study before any interventions were in place. All subjects provided written informed consent.
The protocol was approved by the institutional review board of the University of Vermont.

\section{Demographic, social and economic characteristics}

Income was recorded in seven ordered self-reported categories from less than US $\$ 15,000$ per year to US $\$ 100,000$ per year or more. Education was also recorded as the highest level completed in seven categories from "Less than 9th Grade" to "Graduate or Professional Degree." We collapsed self-reported race and ethnicity into two categories: Non-Hispanic white and all others. Marital status was collapsed into two categories: Married or living as married $v$ s. all others (single, widowed, divorced or separated). We recorded the presence or absence of four types of health insurance: private (commercial indemnity or health maintenance organization benefits often supplied by an employer), Medicare (government health coverage for the elderly and disabled), Medicaid (government health coverage for low income patients), and military (including active duty or veteran's benefits). Subjects may have more than one insurance type.

The shortest driving distance from the patients' homes to their site of care was calculated in kilometers using ArcView 3.3 by Environmental Systems Research Institute, Inc., and a geographic data set purchased from TeleAtlas, Inc. Driving distance was defined as the shortest distance along roads and highways [8].

\section{Physical characteristics}

Height was measured using a portable stadiometer (SECA, Inc.), weight with a portable scale (LB Dial Scale HAP200KD-41, Healthometer, Inc.), and blood pressure with an automated sphygmomanometer (Omron Model HEM-711). Blood pressure was obtained in the seated position in the left arm (unless contraindicated), using the cuff size recommended by the manufacturer. Three readings were obtained at 5-minute intervals and were averaged for the final result. Body mass index was calculated as weight in kilograms divided by height in meters squared.

\section{Laboratory results}

Glycosolated hemoglobin A1C was measured at 13 clinical laboratories in the patients' home communities. All laboratories used the same high-pressure liquid chromatography method with identical reference ranges. Serum creatinine, urine microalbumin-to-creatinine ratio, total cholesterol, high density lipoprotein cholesterol, and triglycerides were likewise measured by the laboratories. Low density lipoprotein cholesterol (LDL) was calculated using the Friedwald formula $(\mathrm{LDL}=$ Total cholesterol high density lipoprotein cholesterol - triglycerides/5) [9] from fasting specimens. Each patient was classified as being above or below certain laboratory value thresholds 
recommended by the American Diabetes Association (A1C >8\%; A1C <7\%; microalbumin-to-creatinine ratio $<30 \mathrm{mg} / \mathrm{mmol}$ ) [10]. If the LDL was $100 \mathrm{mg} / \mathrm{dl}$ or greater, or if it could not be calculated because the triglycerides were above $400 \mathrm{mg} / \mathrm{dl}$, we categorized lipids as above goal. Tests were ordered by the primary care provider when clinically indicated. We report the most recent laboratory assays done before the home visit.

\section{Quality of care}

Where possible, we classified each subject as meeting or not meeting recommendations for care made by the American Diabetes Association [10] and the Vermont Program for Quality in Health Care [11]. Creatinine and urine microalbumin tests were due every year. A1C was on time if the latest test was within 3 months ( 6 months if the latest result was $<7.0 \%$ ). Lipid testing was on time if the latest test was within 6 months (12 months if the latest result showed LDL-cholesterol under $100 \mathrm{mg} / \mathrm{dl}$ ). Additional measures indicate if the subject was both on time and had results on target for A1C and LDL. Pneumonia vaccine was recommended once ever. Influenza vaccine was considered up to date if the patient reported it was given in the current or previous calendar year.

\section{Health habits}

Alcohol consumption was measured by asking: "How many drinks of the following alcoholic beverages do you have in a typical week (including weekends)?

Bottles or cans of beer:

Glasses of wine or wine coolers:

Mixed drinks or shots of liquor:

Subjects who indicated that they do not currently drink alcohol were assigned zero to each of the three beverage categories. A summary variable representing total consumption was constructed as the sum of the three beverage-specific responses. Subjects were also asked the four CAGE screening questions [12].

Tobacco use was assessed by asking: "Have you smoked a cigarette - even one puff - during the past seven days?" Those responding "yes" were asked "How many cigarettes do you smoke on an average day?"

\section{Self care}

We assessed self-care behavior with the Summary of Diabetes Self Care Activities Measure [13]. This instrument asks the subject to record how many days in the last week they performed recommended self-care activities such as following a healthful eating plan, or participating in at least 30 minutes of physical activity. Eleven items are used to generate 5 summary scores representing the fraction of days the subject performs recommended activities related to general diet, diabetes-specific diet, exercise, blood glucose self-monitoring, and foot care. Each score ranges from 0 to 100 .

\section{Literacy}

The Short Test of Functional Health Literacy in Adults (STOFHLA) is a 7-minute timed instrument that measures the ability to read health-related material $[14,15]$. The score ranges from 0 to 36 items answered correctly. Responses can be categorized at "inadequate" (STOFHLA 0-16), "marginal" (STOFHLA 17-22), and "adequate" (STOFHLA 23-36).

\section{Comorbidity}

The Self-Administered Comorbidity Questionnaire is a modification of the widely used Charlson Index. It uses patient interview or questionnaire responses rather than chart abstraction for assessment of comorbidity and has excellent agreement with the chart-based Charlson Index $[16,17]$. We calculated the rate of endorsement of each of 18 specific conditions as well the number of conditions endorsed. We also calculated a score with one point if the condition is endorsed and additional points if the subject reports currently receiving treatment for it, or if it limits activities. Each condition may, therefore, contribute 0 to 3 points for a possible maximum of 54 points. One of the conditions, "eye, nerve, or kidney damage due to diabetes" may be considered a complication of diabetes rather than strictly a comorbidity.

\section{Functional status and depression}

The Medical Outcomes Trust SF-12 Health Survey is a widely used, validated instrument for assessment of general (rather than disease-specific) functional status [18]. Two summary scales are calculated: the Physical Component Summary and the Mental Component Summary. The Patient Health Questionnaire-9 is a brief self-report instrument that quantifies the presence and degree of mental depression [19].

\section{Complications}

We assessed the presence of diabetes complications by asking six questions. The responses were "Yes, " "No," and "Don't know."

1. Have you ever had an ulcer or sore on your leg or foot that took more than 4 weeks to heal?

Has your doctor or health care provider ever told you that you have these problems:

2. Problems with vision or retinopathy related to your diabetes? 
3. Pain, burning, or numbness in the feet or legs related to your diabetes?

4. Problems with stomach emptying related to your diabetes?

5. Problems with sexual function?"

6. Problems with your kidneys related to your diabetes?

\section{Medications}

The subjects were asked to produce "all medications you have used in the past month including prescriptions, overthe-counter products, vitamins, and herbs." The field assistant recorded the name, strength, dose, route, and frequency of each preparation.

\section{Quality of life}

The Audit of Diabetes-Dependant Quality of Life is an 18item questionnaire regarding the impact of diabetes on specific aspects of a person's life with patient weighting of the impact of each domain $[20,21]$. We employed 17 of the 18 domains of this instrument. The scores for each domain can range from -9 (maximum negative impact of diabetes on that domain) to +9 (maximum positive impact).

\section{Resource utilization}

The survey included items asking the subjects to record whether they had used various services in the last year: Endocrinologist, Dietician, Podiatrist, Diabetes Educator, Ophthalmologist, and Diabetes Class. Those answering "Don't Know" were assigned a value of "No." It also prompted subjects to report the number of Emergency Room visits they had in the last year and "In the past month, how many times have you been to a doctor or health care professional?"

\section{Statistical analyses}

In the random effects model, the ICC is the proportion of the total variance that is between clusters (practices).

$I C C=\sigma_{b}^{2} /\left(\sigma_{b}^{2}+\sigma_{w}^{2}\right)$

where $\sigma_{b}^{2}$ is the between-cluster component of variance while $\sigma_{w}^{2}$ is the within-cluster component. If a measurement varies across patients without regard to which practice they are in, the ICC will be close to zero. If the value of the variable is largely a function of which practice they are in, the ICC will be close to 1.0 [2]. We used the analysis of variance estimator [22-24] provided by the "loneway" command in STATA 8.2 (Stata Corp., College
Station, Texas). This estimator uses the F statistic to calculate the ICC for $\mathrm{N}$ total subjects in $\mathrm{k}$ groups of size $\mathrm{N}_{\mathrm{o}}$ :

$$
I C C=\frac{F_{o b s}-1}{F_{o b s}-1+g}
$$

where

$g=\frac{N-\sum_{i} N_{o}^{2} / N}{k-1}$.

Further,

$\sqrt{(V)(I C C)}$

is the asymptomatic standard error of the ICC, and the $100(1-\alpha) \%$ confidence interval is:

$I C C \pm z_{\alpha / 2} \sqrt{(V)(I C C)}$.

For each characteristic, we recorded the sample size $(\mathrm{N})$, the sample size per cluster $\left(\mathrm{N}_{\mathrm{o}}\right)$ the mean (or proportion for dichotomous variables), the standard deviation (SD) for continuous variables, the standard error of the mean (or percentage) adjusted for clustering within practices (SE), the ICC, and the 95\% confidence interval of the ICC. We assessed the association between the value (reported proportion) of binary variables and the ICC [25] with Spearman's non-parametric correlation coefficient. For proportions greater than 0.5 , we used the complement of the proportion so that all proportions for this analysis were less than 0.5. To compare groups of ICCs, we used the two-sample Wilcoxon rank-sum (Mann-Whitney) test.

\section{Results}

The results appear in Table 1 . The 112 ICCs ranged from 0 for 15 variables with negative values truncated at zero to 0.288 for the proportion with a creatinine measurement on time. The median value was 0.0185 with an inter-quartile range of $(0.006,0.037)$. Results were similar for 62 binary variables (median 0.022; IQR 0.006, 0.040) and 50 continuous variables (median 0.017; IQR 0.006, 0.032). A Wilcoxon rank-sum test gave a $P$ value of 0.54 for the comparison between ICCs of continuous and binary variables.

The ICCs for the 62 binary variables were significantly associated with their proportions (Spearman's correlation coefficient $=0.53 ; P<0.0001)$ to a degree sometimes classified as "Large" [26].

\section{Discussion}

These data provide estimates of intra-cluster correlations for 112 patient characteristics relevant to the analysis of 
Table I: Descriptive statistics and intra-practice correlation coefficients

\begin{tabular}{llllllll}
\hline Variable & $\mathrm{N}$ & $\mathrm{N}_{\circ}$ & Mean or percent & SD SE ICC & $95 \%$ confidence interval
\end{tabular}

Demographic, social and economic characteristics

\section{Sex (\% male)}

Age (years)

Married (\%)

High School Graduate (\%)

Income $<\$ 30,000$ per year $(\%)$

Private insurance (\%)

Medicare (\%)

Medicaid (\%)

Military or veterans' insurance (\%)

No insurance (\%)

Travel distance from home to Primary Care provider $(\mathrm{km})$

Non-Hispanic white (\%)

\section{Physical characteristics}

Heart Rate (beats per min)

Systolic blood pressure $(\mathrm{mmHg})$

Diastolic blood pressure $(\mathrm{mmHg})$

Blood pressure below $130 / 80 \mathrm{mmHg}(\%)$

Blood pressure over $140 / 90 \mathrm{mmHg}(\%)$

Height $(\mathrm{cm})$

Weight $(\mathrm{kg})$

Body Mass Index $\left(\mathrm{kg} / \mathrm{m}^{2}\right)$

Body Mass Index $>30 \mathrm{~kg} / \mathrm{m}^{2}(\%)$

\section{Laboratory results}

Mean glycosolated hemoglobin (AIC) (\%)

AIC $>8.0 \%(\%)$

AIC $<7.0(\%)$

Total cholesterol $(\mathrm{mg} / \mathrm{dl})$

LDL-cholesterol $(\mathrm{mg} / \mathrm{dl})$

$\mathrm{LDL}<100 \mathrm{mg} / \mathrm{dl}(\%) *$

Triglycerides ( $\mathrm{mg} / \mathrm{dl})$

Serum creatinine $(\mathrm{mg} / \mathrm{dl})$

Urine microalbumin to creatinine ratio $(\mathrm{mg} / \mathrm{mmol})$

Urine microalbumin to creatinine ratio $<30 \mathrm{mg} / \mathrm{mmol}$ (\%)

\section{$\mathrm{N}$}

(2)

19.7

$\begin{array}{ll}8808 & 119.7 \\ 8286 & 117.3\end{array}$

$\begin{array}{ll}8286 & 117.3 \\ 1004 & 14.5\end{array}$

$999 \quad 14.4$

$931 \quad 13.4$

$1001 \quad 14.4$

$997 \quad 14.4$

$995 \quad 14.3$

$994 \quad 14.3$

$993 \quad 14.3$

$2955 \quad 76.5$

$1004 \quad 14.5$

\begin{tabular}{|c|c|c|c|c|}
\hline 48.4 & & 1.3 & 0.038 & $0.021,0.056$ \\
\hline 63.1 & 13.9 & 0.5 & 0.077 & $0.045,0.109$ \\
\hline 62.5 & & 1.6 & 0.009 & $0,0.036$ \\
\hline 75.4 & & 1.6 & 0.011 & $0,0.038$ \\
\hline 59.0 & & 2.1 & 0.042 & $0.003,0.081$ \\
\hline 58.4 & & 2.0 & 0.050 & $0.011,0.090$ \\
\hline 59.9 & & 1.7 & 0.018 & $0,0.047$ \\
\hline 21.4 & & 1.8 & 0.036 & $0,0.071$ \\
\hline 5.2 & & 0.8 & 0.029 & $0,0.062$ \\
\hline 2.4 & & 0.6 & 0.035 & $0,0.070$ \\
\hline 13.7 & 15.3 & 1.8 & 0.196 & $0.102,0.289$ \\
\hline 97.3 & & 0.5 & 0.003 & $0,0.028$ \\
\hline
\end{tabular}

$\begin{array}{llc}998 & 14.4 & 75.0 \\ 999 & 14.4 & 140.3 \\ 999 & 14.4 & 78.3 \\ 999 & 14.4 & 25.0 \\ 999 & 14.4 & 49.2 \\ 998 & 14.4 & 165.2 \\ 997 & 14.4 & 203.0 \\ 994 & 14.3 & 33.8 \\ 994 & 14.3 & 67.2\end{array}$

$\begin{array}{cccc}13.0 & 0.5 & 0.015 & 0,0.043 \\ 19.6 & 0.8 & 0.042 & 0.005,0.079 \\ 10.5 & 0.4 & 0.017 & 0,0.047 \\ & 1.6 & 0.028 & 0,0.061 \\ & 2.3 & 0.069 & 0.024,0.114 \\ 10.4 & 0.4 & 0.019 & 0,0.048 \\ 47.7 & 1.6 & 0.011 & 0,0.038 \\ 7.4 & 0.2 & 0.011 & 0,0.039 \\ & 1.6 & 0.010 & 0,0.038\end{array}$

$\begin{array}{llc}8711 & 118.4 & 7.01 \\ 8711 & 118.4 & 18.1 \\ 8711 & 118.4 & 61.7 \\ 8167 & 112.5 & 184.4 \\ 7834 & 111.0 & 105.8 \\ 7873 & 111.6 & 43.8 \\ 7969 & 109.8 & 190.5 \\ 8474 & 115.1 & 1.12 \\ 3039 & 46.7 & 47.0 \\ 3338 & 49.0 & 67.3\end{array}$

$\begin{array}{ccc}1.45 & 0.05 & 0.055 \\ & 1.0 & 0.025 \\ & 1.6 & 0.046 \\ 41.8 & 1.2 & 0.037 \\ 34.2 & 1.0 & 0.045 \\ & 1.4 & 0.029 \\ 161.3 & 3.3 & 0.014 \\ 0.70 & 0.02 & 0.080 \\ 260.7 & 10.0 & 0.101 \\ & 1.5 & 0.031\end{array}$

$0.032,0.079$

$0.013,0.037$

$0.026,0.066$

$0.020,0.054$

$0.025,0.065$

$0.020,0.055$

$0.006,0.022$

$0.048,0.112$

$0.051,0.152$

$0.011,0.051$ 


\section{Quality of care (Process Measures)}

Creatinine on time (\%)

Urine microalbumin to creatinine ratio on time (\%)

AIC on time (\%)

Lipids on time $(\%)$

AIC on time $\&<7 \%(\%)$

LDL on time \& $<100 \mathrm{mg} / \mathrm{dl}(\%)$

Influenza vaccine given (\%)

Pneumonia vaccine given (\%)

$\begin{array}{ccc}8808 & 119.7 & 79.4 \\ 8808 & 119.7 & 24.6 \\ 8808 & 119.7 & 50.7 \\ 8808 & 119.7 & 70.3 \\ 7308 & 113.2 & 35.5 \\ 7308 & 113.2 & 3 \\ 995 & 14.3 & 80.5 \\ 913 & 13.2 & 70.6\end{array}$

79.4
24.6
50.7
70.3
35.5
31.3
80.5
70.6

$0.203,0.373$

$0.105,0.219$

$0.074,0.162$

$0.132,0.265$

$0.021,0.060$

$0.021,0.059$

$0.016,0.100$

$0.014,0.102$

\section{Health habits}

Alcoholic drinks per week

CAGE score (0-4)

Tobacco smoker (\%)

Cigarettes per day

$\begin{array}{cc}988 & 14.2 \\ 946 & 13.6 \\ 1006 & 14.5 \\ 159 & 2.6\end{array}$

14.2
13.6
14.5
2.6

1.6

Summary of Diabetes Self Care Activities Measure

General diet score

Specific diet score

Exercise score

Blood glucose self-monitoring score

0.2

6.9

17.2

$\begin{array}{lll}4.5 & 0.2 & 0.027 \\ 0.7 & 0.2 & 0.009 \\ & 1.2 & 0.005 \\ 11.2 & 1.0 & 0.086\end{array}$

$0,0.060$

$0,0.038$

$0,0.030$

Foot care score

$\begin{array}{lll}953 & 13.7 & 58.4 \\ 980 & 14.1 & 52 . \\ 992 & 14.3 & 34.6 \\ 933 & 13.5 & 57.9 \\ 990 & 14.3 & 44 .\end{array}$

58.4
52.2
34.6
57.9
44.2

$\begin{array}{lll}33.3 & 1.2 & 0.017 \\ 23.9 & 0.8 & 0.01 \\ 32.7 & 1.2 & 0.02 \\ 38.9 & 1.5 & 0.05 \\ 35.5 & 1.2 & 0.002\end{array}$

\section{Literacy: Short Test of Functional Health Literacy in Adults}

Test score (0-36 points)

Inadequate $(0-16)(\%)$

Adequate (23-36) (\%)

$\begin{array}{lll}1002 & 14.4 & 29.7 \\ 1002 & 14.4 & 10.5 \\ 1002 & 14.4 & 82\end{array}$

\section{Comorbidity}

Congestive heart failure (\%)

Coronary artery disease (\%)

Peripheral vascular disease (\%)

Stroke (\%)

Dementia (\%) $†$

Asthma (\%)

Arthritis (\%)

Peptic ulcer disease (\%)

Cirrhosis (\%)

$\begin{array}{ccc}1002 & 14.4 & 29.7 \\ 1002 & 14.4 & 10.5 \\ 1002 & 14.4 & 82.9 \\ & & \\ 1006 & 14.5 & 17.1 \\ 1006 & 14.5 & 19.3 \\ 1006 & 14.5 & 8.7 \\ 1006 & 14.5 & 11.7 \\ 1006 & 14.5 & 0.9 \\ 1006 & 14.5 & 20.2 \\ 1006 & 14.5 & 14.1 \\ 1006 & 14.5 & 14.3 \\ 1006 & 14.5 & 1.9\end{array}$

9.6

$\begin{array}{ll}0.3 & 0.017 \\ 1.0 & 0.011 \\ 1.5 & 0.037\end{array}$

$0,0.079$

0.039 
Table I: Descriptive statistics and intra-practice correlation coefficients (Continued)

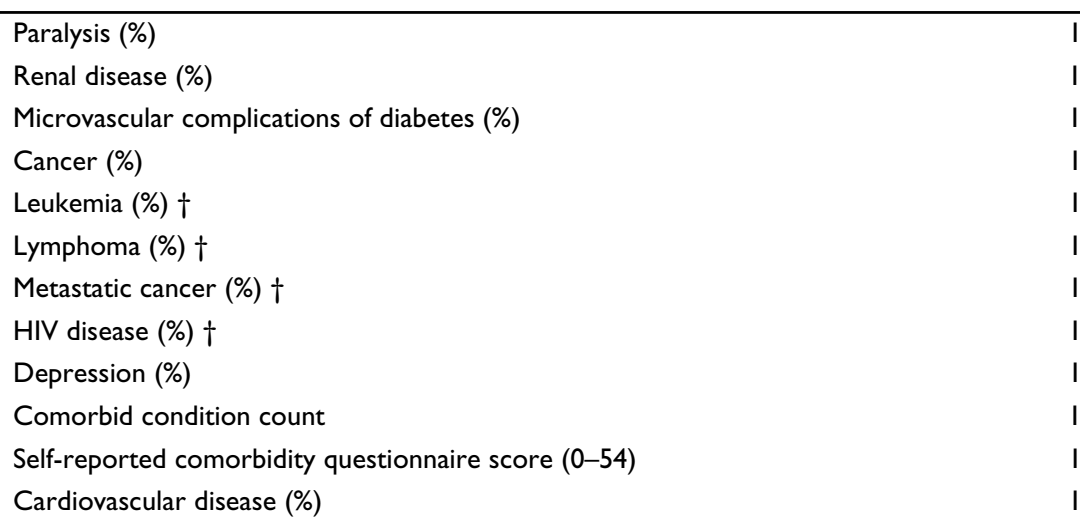

\begin{tabular}{|c|c|c|c|c|c|}
\hline 1006 & 3.0 & & 0.6 & 0.006 & $0,0.032$ \\
\hline 1006 & 5.0 & & 0.6 & $0 \S$ & $0,0.024$ \\
\hline 1006 & 16.9 & & 1.2 & 0.012 & $0,0.040$ \\
\hline 1006 & 11.9 & & 1.0 & $0 \S$ & $0,0.024$ \\
\hline 1006 & 0.6 & & 0.2 & $0 \S$ & $0,0.024$ \\
\hline 1006 & 0.5 & & 0.3 & 0.018 & $0,0.048$ \\
\hline 1006 & 0.7 & & 0.3 & $0 \S$ & $0,0.024$ \\
\hline 1006 & 0.1 & & 0.1 & $0 \S$ & $0,0.024$ \\
\hline 1006 & 35.0 & & 1.7 & 0.007 & $0,0.033$ \\
\hline 1006 & 1.8 & 1.7 & 0.1 & 0.031 & $0,0.065$ \\
\hline 1006 & 3.6 & 3.9 & 0.2 & 0.032 & $0,0.066$ \\
\hline 1006 & 30.9 & & 1.6 & 0.018 & $0,0.048$ \\
\hline
\end{tabular}

Functional status

SF-12 Physical Component Summary score (0-100)

SF-12 Mental Component Summary score (0-100)

Patient Health Questionnaire-9 depression score (0-27)

$986 \quad 14.2$

$986 \quad 14.2$

$591 \quad 11.7$

41.2
50.0

50.0

4.1

$\begin{array}{lll}12.4 & 0.5 & 0.028 \\ 10.7 & 0.4 & 0.032 \\ 5.0 & 0.2 & 0.006\end{array}$

0.006
$0,0.062$

$0,0.061$

$0,0.044$

\section{Complications of diabetes}

Foot ulcer (\%)

Vision problems (\%)

Foot or leg pain (\%)

Stomach emptying problems (\%)

Sexual problems (\%)

Kidney problems $(\%)$

$992 \quad 14.3$

$950 \quad 13.7$

$943 \quad 13.6$

$901 \quad 13.0$

$923 \quad 13.3$

$490 \quad 10.3$

\section{Medications}

Insulin (\%)

Oral hypoglycemic agent (\%)

Total medication count (0-29)

Prescription medication count (0-24)

Non-prescription medication count (0-15)

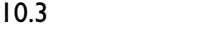

11.3
20.1
30.9
6.2
26.4
9.2

$\begin{array}{cc}1.0 & 0.009 \\ 1.3 & 0 \\ 1.8 & 0.023 \\ 0.8 & 0.003 \\ 1.4 & 0 \\ 1.4 & 0.040\end{array}$

$\begin{array}{cc}0.009 & 0,0.036 \\ 0 \S & 0,0.025 \\ 0.023 & 0,0.055 \\ 0.003 & 0,0.030 \\ 0 \S & 0,0.026 \\ 0.040 & 0,0.096\end{array}$

\section{Audit of Diabetes-Dependant Quality of Life}

Do physically

Confidence in ability

Motivation

Freedom to eat

Enjoyment of food

$\begin{array}{ll}1006 & 14.5 \\ 1006 & 14.5 \\ 1006 & 14.5 \\ 1006 & 14.5 \\ 1006 & 14.5\end{array}$

$\begin{array}{ccccc}18.6 & & 1.4 & 0.014 & 0,0.042 \\ 66.6 & & 1.7 & 0.022 & 0,0.053 \\ 8.8 & 4.5 & 0.2 & 0.028 & 0,0.061 \\ 6.7 & 3.8 & 0.2 & 0.055 & 0.014,0.095 \\ 2.0 & 2.4 & 0.1 & 0.059 & 0.017,0.101\end{array}$

$\begin{array}{ll}985 & 14.2 \\ 982 & 14.2 \\ 981 & 14.2 \\ 985 & 14.2 \\ 986 & 14.2\end{array}$

-1.9
-2.0
-1.9
-2.8
-2.5

$\begin{array}{ccc}2.6 & 0.1 & 0.001 \\ 2.7 & 0.1 & 0.004 \\ 2.7 & 0.1 & 0 \S \\ 2.9 & 0.1 & <0.001 \\ 2.9 & 0.1 & 0 \S\end{array}$


Table I: Descriptive statistics and intra-practice correlation coefficients (Continued)

\begin{tabular}{ll}
\hline Future & \\
Freedom to drink & \\
Dependence & \\
Working life & \\
Family life & \\
Social life & \\
Sex life & Physical appearance \\
Holidays/Leisure & Travel \\
Society reaction & Finances \\
Average weighted impact
\end{tabular}

$984 \quad 14.2 \quad-0.6$

$-0.6$

3.8

$0.10 \S$

$0 \S$

$0,0.025$

14.2

14.2

$-1.5$

$-0.6$

2.4

0.1
0.1
0.1

14.2
14.2

$-1.5$

14.2

14.2

14.1

14.1
14.1

14.2

14.I

14.1

14.1

14.3

$-0.6$

$-0.9$

$-1.0$

$-1.1$

$-1.5$

$-1.6$

$-0.5$

$-1.7$

$-1.5$

$\begin{array}{lll}2.3 & 0.1 & 0.014 \\ 2.7 & 0.1 & 0.035\end{array}$

$\begin{array}{lll}2.0 & 0.1 & 0 \S\end{array}$

$\begin{array}{lll}2.4 & 0.1 & 0.020\end{array}$

$\begin{array}{lll}2.4 & 0.1 & 0.020 \\ 2.3 & 0.1 & 0.012\end{array}$

$\begin{array}{lll}2.5 & 0.1 & 0.1\end{array}$

$\begin{array}{lll}2.6 & 0.1 & 0.022\end{array}$

$\begin{array}{lll}1.6 & 0.1 & 0.026\end{array}$

$\begin{array}{lll}2.7 & 0.1 & 0.009\end{array}$

$\begin{array}{lll}2.0 & 0.1 & 0.004\end{array}$

$0,0.025$

$0,0.042$

$0,0.070$

$0,0.025$

$0,0.051$

\section{$0,0.041$}

$0,0.025$

$0,0.054$

$0,0.058$

$0,0.037$

$0,0.029$

\section{Resource utilization}

Endocrinologist in last year (\%)

Dietician in last year (\%)

Podiatrist in last year (\%)

Diabetes Educator in last year (\%)

Ophthalmologist in last year (\%)

Diabetes class in last year (\%)

Emergency Room visits in last year (mean)

Health professional visits in last month (mean)

$\begin{array}{ll}971 & 14.0 \\ 984 & 14.2 \\ 984 & 14.2 \\ 970 & 14.0 \\ 944 & 13.6 \\ 999 & 14.4 \\ 994 & 14.3 \\ 991 & 14.3\end{array}$

15.9
34.3
23.8
23.8
60.4
35.0
0.64
1.63

$\begin{array}{ccc} & 2.0 & 0.089 \\ & 1.8 & 0.018 \\ & 1.6 & 0.022 \\ & 1.8 & 0.041 \\ & 2.1 & 0.041 \\ & 1.9 & 0.035 \\ 1.46 & 0.05 & 0.015 \\ 2.39 & 0.08 & 0.035\end{array}$

$0.037,0.140$
$0,0.048$
$0,0.054$
$0.004,0.078$
$0.003,0.079$
$0.002,0.073$
$0,0.044$
$0,0.070$

$\mathrm{N}=$ Subjects analyzed; $\mathrm{N}_{\mathrm{o}}=$ Number per cluster; $\mathrm{SD}=$ standard deviation of mean; $\mathrm{SE}=$ standard error adjusted for clustering; ICC = Intra-cluster (intra-practice) correlation coefficient. *In 39 cases in which LDL could not be estimated because triglycerides were over $400 \mathrm{mg} / \mathrm{dl}$, LDL was assumed to be over $100 \mathrm{mg} / \mathrm{dl}$.

$\dagger$ Because only a few subjects have this condition, the ICC may not be estimated consistently.

$\S$ The method used truncates the estimate of ICC (and its $\mathrm{Cl}$ ) at 0 for some values that may be negative. 
adults with diabetes receiving primary care in Vermont and nearby regions of the United States. They may usefully be applied to the design and sample size estimation of future surveys that are clustered on primary care practices.

In the design of clustered-based studies, the ICC may be used to calculate the design effect which is the degree to which the sample size must be inflated above that of a simple random sample to account for the loss of information inherent in the clustered design. If the average number of subjects sampled per cluster is $m$, the design effect is given by:

Design effect $=1+(m-1) \cdot \mathrm{ICC}$

If $m$ or ICC is large, the total number of individual subjects needed may be substantially greater than suggested by a sample size calculation that is not adjusted for clustering. Alternatively, if both $m$ and ICC are small, the design effect may be very close to 1.0 indicating that the clustered design does not inflate the sample size.

In the VDIS, the cost of enrolling subjects for laboratory data collection within a cluster, once the practice was enrolled, was relatively low. Therefore, large values of $m$ (120.7 subjects per practice on average) were not problematic. However, the cost per patient of obtaining interview data was relatively high. Therefore, we reduced the mean sample size per cluster to 14.5 by random sampling within practice. The design effects experienced in VDIS are not representative of those faced in other designs unless they happen to have the same mean cluster sample size as VDIS (which is extremely unlikely). Therefore, unlike previous publications, we have elected not to report design effects. Study designers should use the ICCs and their own estimates of $m$ to understand their own design effects.

Campbell et al [27] suggest that ICCs are higher for process measures than for outcomes. We see evidence for this in that the eight quality of care variables (process measures, see Table 1) have a median ICC of 0.088 (IQR 0.049, 0.181 ) while the ten laboratory outcomes measures have a median ICC of 0.038 (IQR 0.029, 0.055). This difference is significant by Wilcoxon rank-sum test with $P=0.013$. We note that the nine physical characteristics of the subjects, presumably under less control of the provider than either laboratory results or even process measures, have a median ICC of 0.017 (IQR 0.011, 0.028) and are significantly different than the laboratory measures $(P=0.034)$.

Within practice correlation was most prominent for process measures associated with quality of care. The likelihood of receiving a creatinine measurement on time had the highest ICC $(0.288)$ with other quality of care meas- ures also among the most highly correlated. This may represent that process measures are heavily influenced by the practice style of the practitioners and any office-based procedures (reminders, registries, flow sheets, etc.) that only some practices employ. In a similar vein, physiologic control of some aspects of diabetes (especially achievement of tight control of A1C and LDL) appears to vary importantly across practices with ICCs of 0.046 for A1C below $7 \%$ and 0.029 for LDL below $100 \mathrm{mg} / \mathrm{dl}$.

Some demographic aspects of the population were correlated within practices: age, sex, and especially travel burden. As patients tend to stay with their primary provider as they age, some practices accumulate older patients (ICC $=0.077)$. Some patients express a preference for same-sex providers, with more women visiting practices that have female providers. This may account for the relatively high ICC for sex (0.038). Travel distance may be related to the geographic location of the practice office. Practices in more densely populated areas may tend to have lower typical travel burdens.

With the possible exception of blood pressure (which may be under the control of the providers to some degree), the physical characteristics and health habits of patients vary little across practices.

Although apparently under the control of the providers, the utilization of health care services had generally small ICCs. The exception was consultation by an Endocrinologist with an ICC of 0.089 . This may reflect the geographic proximity of an endocrinologist to some of the practices.

The aspects of diabetes that are most directly experienced by patients (complications, quality of life, functional status, comorbidity, and self-care) vary little across practices. It does not appear that some primary care practices tend to accumulate more complicated or difficult patients than others. Likewise, low health literacy is a substantial, and perhaps unrecognized, problem for all practices, with little clustering within practices.

These data demonstrate a large correlation between the proportion of the 62 binary variables and their ICCs. This finding has been noted by others [25] and may be useful in estimating an ICC for sample size calculations.

For many of these variables, the impact of within-cluster correlation on sample size requirements appears to be relatively small. Thirty-three variables had an ICC $<0.010$ with a design effect less than 1.14 indicating that the VDIS clustered design required an increase in sample size of $14 \%$ compared to a non-clustered study. However, depending on the number of clusters and the number of 
subjects per cluster, even small ICCs can result in the need for costly increases in overall sample size.

These estimates of ICC are not useful for studies that cluster on factors other than practice (such as community, hospital, individual provider, family, classroom, etc.). The VDIS study population was drawn from Vermont and nearby New York and New Hampshire and is, therefore, predominantly white and rural. All the subjects were under care for diabetes. Although some have Type 1 diabetes, this older, overweight population is largely comprised of patients with Type 2 diabetes. The practices in the VDIS are small with a median of 2 providers per practice. For these reasons, generalization to other populations and settings may be problematic.

Several recent reports provide some comparisons from other settings (Table 2). A study of British patients aged 75 and older reported intra-practice correlations from 106 general practices [4]. A study from Rhode Island and nearby Massachusetts enrolled 15 primary care practices [5]. Several surveys of general practices from Australia and New Zealand provided a few ICCs comparable to VDIS $[3,6]$. The ICCs for most of these variables vary substantially across the studies. For instance, the ICCs for weight and body mass index varied between 0.011 and 0.081 . Differences in the practice structures, referral patterns, social and geographic factors and practice patterns may explain these differences. Only recently have determinants of ICC begun to be studied [26-28]. We suggest that more catalogues of ICCs, drawn from a variety of settings, will be useful both to investigators designing new clustered studies and to researchers investigating the role of setting on patient characteristics.

\section{Conclusion}

Intra-practice correlation coefficients in this survey of adults receiving care for diabetes varied widely. Some characteristics (such as the likelihood of having a recent creatinine measurement) were highly associated with the practice (ICC $=0.288$ ), while others (prevalence of some comorbidities and complications and certain aspects of quality of life) varied much more across patients with virtually no correlation within practices (ICC $<0.001$ ). The values reported here may be useful in designing future clustered studies.

\section{Competing interests}

The author(s) declare that they have no competing interests.

\section{Authors' contributions}

BL conceptualized the research and performed the analyses. CDM oversaw the data collection and management. Both authors read and approved the final manuscript.

\section{Acknowledgements}

This work was funded by the National Institute of Diabetes and Digestive and Kidney Diseases (ROI DK6II 67 and K24 DK068380).

\section{References}

I. Kerry SM, Bland JM: Analysis of a trial randomized in clusters. BMJ 1998, 3 I 6:54.

2. Kerry SM, Bland JM: The intracluster correlation coefficient in cluster randomization. $B M J$ I998, 3 I 6: 1455.

3. Knox SA, Chondros P: Observed intra-cluster correlation coefficients in a cluster survey sample of patient encounters in general practice in Australia. BMC Medical Research Methodology 2004, 4:30.

4. Smeeth L, Ng ES: Intraclass correlation coefficients for cluster randomized trials in primary care: data from the MRC Trial of the Assessment and Management of Older People in the Community. Controlled Clinical Trials 2002, 23:409-21.

5. Parker DR, Evangelou E, Eaton CB: Intraclass correlation coefficients for cluster randomized trials in primary care: the cholesterol education and research trial (CEART). Contemporary Clinical Trials 2005, 26:260-7.

6. Elley CR, Kerse N, Chondros P, Robinson E: Intraclass correlation coefficients from three cluster randomised trials in primary and residential care. Aust N ZJ Public Health 2005, 29:46I-467.

7. MacLean CD, Littenberg B, Gagnon M, Reardon M, Turner P, Jordan $C$ : The Vermont Diabetes Information System (VDIS): study design and subject recruitment for a cluster randomized trial of a diabetes registry in a statewide sample of primary care practices. Clinical Trials 2004, I:532-544.

8. Strauss K, MacLean CD, Troy AR, Littenberg B: Driving distance as a barrier to glycemic control in diabetes. J Gen Intern Med 2006, 21:378-80.

9. Friedwald WT, Levy RI, Frederickson DS: Estimation of the concentration of low density lipoprotein cholesterol in plasma, without use of the preparative ultracentrifugation. Clin Chem 1972, 18:499-502.

10. American Diabetes Association: Clinical Practice Recommendations. Diabetes Care 2005, 28(SuppI I): [http://care.diabetesjour nals.org/cgi/content/full/28/suppl $\mid / /$ I $]$.

II. Vermont Program for Quality in Health Care: Recommendations for management of Diabetes in Vermont. 2004 [http:// www.vpghc.org/CurrentReports/Diabetes\%202004.pdf]. Montpelier: Vermont Program for Quality in Health Care

12. Ewing JA: Detecting Alcoholism: The CAGE Questionnaire. JAMA 1984, 252: 1905- 1907.

13. Toobert DJ, Hampson SE, Glasgow RE: The summary of diabetes self-care activities measure: results from 7 studies and a revised scale. Diabetes Care 2000, 23:943-50.

14. Parker RM, Baker DW, Williams MV, Nurss JR: The test of functional health literacy in adults: a new instrument for measuring patients' literacy skills. J Gen Intern Med 1995, 10:537-4I.

15. Baker DW, Williams MV, Parker RM, Gazmararian JA, Nurss J: Development of a brief test to measure functional health literacy. Patient Educ Couns 1999, 38:33-42.

16. Katz JN, Chang LC, Sangha O, Fossel AH, Bates DW: Can comorbidity be measured by questionnaire rather than medical record review? Med Care 1996, 34:73-84.

17. Sangha O, Stucki G, Liang MH, Fossel AH, Katz JN: The Self-Administered Comorbidity Questionnaire: a new method to assess comorbidity for clinical and health services research. Arthritis Rheum 2003, 49:156-63.

18. Ware JE, Kosinski M, Turner-Bowker DM, Gandek B: How to Score Version 2 of the SF-I2 Health Survey Lincoln, RI: Quality Metric Inc; 2002.

19. Kroenke K, Spitzer RL, Williams JB: The PHQ-9: validity of a brief depression severity measure. J Gen Intern Med 200I, I 6:606-13.

20. Bradley C, Todd C, Gorton T, Symonds E, Martin A, Plowright R: The development of an individualized questionnaire measure of perceived impact of diabetes on quality of life: the ADDQoL. Qual Life Res 1999, 8:79-91.

21. Bradley C, Speight J: Patient perceptions of diabetes and diabetes therapy: assessing quality of life. Diabetes Metab Res Rev 2002, I8(Suppl 3):S64-9. 
22. Stata Corporation: Loneway - Large one-way ANOVA random effects, and reliabaility. In Stata Base Reference Manual, G-M, Release 8 Volume 2. College Station, TX: Stata Press; 2003:338-43.

23. Gleason JR: Computing intraclass correlations and large ANOVAs. Stata Technical Bulletin 1997, 35:25-31.

24. Donner A: A review of inference procedures for the intraclass correlation coefficient in the one-way random effects model. International Statistical Review 1986, 54:67-82.

25. Gulliford MC, Adams G, Ukoumunne OC, Latinovic R, Chinn S, Campbell MJ: Intraclass correlation coefficient and outcome prevalence are associated in clustered binary data. Journal of Clinical Epidemiology 2005, 58:246-25I.

26. Cohen J: Statistical power analysis for the behavioral sciences 2 nd edition Hillsdale, NJ : Lawrence Erlbaum Associates; 1988.

27. Campbell MK, Fayers PM, Grimshaw JM: Determinants of the intracluster correlation coefficient in cluster randomized trials: the case of implementation research. Clin Trials 2005, 2(2):99-107.

28. Adams G, Gulliford MC, Ukoumunne OC, Eldridge S, Chinn S, Campbell MJ: Patterns of intra-cluster correlation from primary care research to inform study design and analysis. Journal of Clinical Epidemiology 2004, 57:785-794.

\section{Pre-publication history}

The pre-publication history for this paper can be accessed here:

http://www.biomedcentral.com/1471-2288/6/20/prepub

Publish with BioMed Central and every scientist can read your work free of charge

"BioMed Central will be the most significant development for disseminating the results of biomedical research in our lifetime. "

Sir Paul Nurse, Cancer Research UK

Your research papers will be:

- available free of charge to the entire biomedical community

- peer reviewed and published immediately upon acceptance

- cited in PubMed and archived on PubMed Central

- yours - you keep the copyright 